Ján Borsík*, Matematický ústav SAV, Grešákova 6, 04001 Košice, Slovakia, e-mail: borsik@linux1.saske.sk

\title{
ON PRODUCTS OF QUASICONTINUOUS FUNCTIONS
}

\begin{abstract}
It is shown that every cliquish function $f$ defined on a pseudometrizable space for which the preimages of the positive and the negative half-axes are simply open sets is the product of two quasicontinuous functions. If moreover $f$ is in the Baire class $\alpha$, then we can take the factors also in the Baire class $\alpha$.
\end{abstract}

\section{Introduction}

In 1985 in [5] Z. Grande showed that there is a cliquish function of a real variable which cannot be written as the finite product of quasicontinuous functions and asked for characterization of such functions. This characterization has been given by T. Natkaniec in 1990 in [10], where it is shown that a function $f: \mathbb{R} \rightarrow \mathbb{R}$ can be factored into a finite product of quasicontinuous functions if and only if it is cliquish and

$(\star)$ each of the sets $f^{-1}((-\infty, 0)), f^{-1}((0, \infty))$ and $f^{-1}(0)$ is simply open.

However, in his representations of such function he used as many as eight quasicontinuous functions. This result is generalized in [2] where it is shown that each real cliquish function defined on a separable metrizable space satisfying the condition $(\star)$ can be written as the product of three quasicontinuous functions. Moreover, it is shown that the condition $(\star)$ can be replaced with "the set $f^{-1}(0)$ is simply open" for a Baire space. Finally, A. Maliszewski in [8] (see also [7]) proved that two quasicontinuous functions are sufficient for a cliquish function $f$ defined on $\mathbb{R}^{n}$ satisfying $(\star)$. In this paper we generalize this result for a cliquish function satisfying $(\star)$ defined on a pseudometrizable space. The proof is quite different from [8].

Key Words: Quasicontinuity, Cliquishness, Products

Mathematical Reviews subject classification: Primary 54C08

Received by the editors September 25, 1996

* Supported by Grant GA-SAV 1228/96 


\section{Preliminaries}

In what follows, $X$ denotes a topological space. For a subset $A$ of $X$ denote by $\mathrm{Cl} A$ and Int $A$ the closure and the interior of $A$, respectively. If $\mathcal{A}$ is a family of sets in $X$, then $\bigcup \mathcal{A}=\bigcup\{A: A \in \mathcal{A}\}$ and $\mathrm{Cl} \mathcal{A}=\{\mathrm{Cl} A: A \in \mathcal{A}\}$. The letters $\mathbb{R}, \mathbb{Q}$ and $\mathbb{N}$ stand for the set of real, rational and natural numbers, respectively.

We recall that a function $f: X \rightarrow \mathbb{R}$ is said to be quasicontinuous (cliquish) at $x \in X$ if for every neighbourhood $U$ of $x$ and every $\varepsilon>0$ there is a nonempty open set $G \subset U$ such that $|f(x)-f(y)|<\varepsilon$ for each $y \in G(|f(y)-f(z)|<\varepsilon$ for each $y, z \in G$ ) (see e.g. [11]). A function is said to be quasicontinuous (cliquish) if it is such at each point. A function $f: X \rightarrow \mathbb{R}$ is simply continuous if $f^{-1}(V)$ is a simply open set in $X$ for each open set $V$ in $\mathbb{R}$. A set $A$ is simply open if it is the union of an open set and a nowhere dense set [1].

Denote by $\mathcal{Q}, \mathcal{S}, \mathcal{B}_{\alpha}$ and $b$ the class of all quasicontinuous, simply continuous, in Baire class $\alpha$ and bounded functions. Finally, let

$$
\begin{aligned}
& \mathcal{H}=\{f: X \rightarrow \mathbb{R} ; f \text { is cliquish and the sets } \\
& \left.f^{-1}((-\infty, 0)) \text { and } f^{-1}((0, \infty)) \text { are simply open }\right\}
\end{aligned}
$$

\section{$3 \quad$ Results}

We shall show that for a pseudometrizable space we have $\mathcal{Q} \cdot \mathcal{Q}=\mathcal{H}$ and $\mathcal{B}_{\alpha} \mathcal{Q} \cdot \mathcal{B}_{\alpha} \mathcal{Q}=\mathcal{B}_{\alpha} \mathcal{H}$. Evidently $\mathcal{Q} \subset \mathcal{H}$ and by [2] $\mathcal{H} \cdot \mathcal{H}=\mathcal{H}$ for an arbitrary topological space $X$. Two following results are proved in $[4](D(f)$ denotes the set of all discontinuity points of $f$ ).

Lemma 3.1. [4, Lemma 3.1] Let $X$ be a pseudometrizable space. Let $F$ be a nonempty nowhere dense closed set and let $G$ be an open set such that $F \subset$ $\mathrm{Cl} G$. Then there is a family $\mathcal{K}=\bigcup_{n} \mathcal{K}_{n}$ of nonempty open subsets of $X$ such that

(i) $\mathrm{Cl} K \subset G \backslash F$ for each $K \in \mathcal{K}$;

(ii) for each $x \in X \backslash F$ there is a neighborhood $V$ of $x$ such that the set $\{K \in \mathcal{K}: V \cap \mathrm{Cl} K \neq \emptyset\}$ has at most one element;

(iii) for each $x \in F$ and for each neighborhood $U$ of $x$ there is a $k \in \mathbb{N}$ such that for each $n \geq k$ there is $K \in \mathcal{K}_{n}$ with $\mathrm{Cl} K \subset U$.

Theorem 3.2. [4, Theorem 4.1] Let $X$ be a pseudometrizable space. Then every cliquish function $f: X \rightarrow \mathbb{R}$ is the sum of two quasicontinuous functions 
$f_{1}$ and $f_{2}$. Moreover, $D\left(f_{1}\right) \cup D\left(f_{2}\right) \subset D(f)$ and if $f$ is bounded (in Baire class $\alpha$ ), then the summands can be taken bounded (in Baire class $\alpha$ ).

Lemma 3.3. Let $X$ be a pseudometrizable space. If $f: X \rightarrow \mathbb{R}$ is a positive (negative) cliquish function then $f$ is the product of two quasicontinuous functions. If moreover $f$ is in Baire class $\alpha$ then we can conclude that the factors belong to Baire class $\alpha$.

Proof. The function $\ln |f|$ is cliquish (and in Baire class $\alpha$ if $f$ is in Baire class $\alpha$ ) and according to Theorem 3.2 there are quasicontinuous functions $g_{1}, g_{2}: X \rightarrow \mathbb{R}$ such that $\ln |f|=g_{1}+g_{2}$. Now we take $f_{1}=\operatorname{sign} f \cdot \exp g_{1}$ and $f_{2}=\exp g_{2}$.

Theorem 3.4. Let $X$ be a pseudometrizable space. Then every function from $\mathcal{H}$ is the product of two quasicontinuous functions. If moreover $f$ is in Baire class $\alpha$ then we can conclude that the factors belong to Baire class $\alpha$.

Proof. Denote $G_{1}=\operatorname{Int} f^{-1}((-\infty, 0)), G_{2}=\operatorname{Int} f^{-1}((0, \infty))$ and $G_{3}=$ Int $f^{-1}(0)$. Then the set $A=X \backslash\left(G_{1} \cup G_{2} \cup G_{3}\right)$ is closed nowhere dense and by Lemma 3.1 there is a family $\mathcal{K}=\bigcup_{n} \mathcal{K}_{n}$ of nonempty open sets in $X$ satisfying (i)-(iii) for $F=A$ and $G=X$. The set $C=\bigcup \mathrm{Cl} \mathcal{K}$ is closed in $X \backslash A$ and therefore sets $G_{j} \backslash C, j \in\{1,2,3\}$, are open.

Let $j \in\{1,2\}$. By Lemma 3.3 there are quasicontinuous functions $t_{1}^{j}, t_{2}^{j}: G_{j} \backslash$ $C \rightarrow \mathbb{R}$ such that $f_{\mid G_{j} \backslash C}=t_{1}^{j} \cdot t_{2}^{j}$. Further by Lemma 3.3 for each $K \in \mathcal{K}$ with $\mathrm{Cl} K \cap G_{j} \neq \emptyset$ there are quasicontinuous functions $g_{1}^{j, K}, g_{2}^{j, K}: \mathrm{Cl} K \cap G_{j} \rightarrow \mathbb{R}$ such that $f_{\mid \mathrm{Cl} K \cap G_{j}}=g_{1}^{j, K} \cdot g_{2}^{j, K}$. Evidently, $g_{i}^{j, K}(x) \neq 0$ for each $x \in \mathrm{Cl} K \cap G_{j}$ and $i \in\{1,2\}$. If $\mathrm{Cl} K \cap G_{j} \neq \emptyset$ choose an arbitrary $a^{j, K} \in K \cap G_{j}$. Let $\mathbb{Q} \backslash\{0\}=\left\{q_{1}, q_{2}, \ldots\right\}$ be a one-to-one sequence of all rationals different from zero. Let $\beta: \mathbb{N} \times \mathbb{N} \rightarrow \mathbb{N}$ be defined by $\beta(m, k)=2^{k-1} \cdot(2 m-1)$, let $\mathbb{N}_{1}$ be the set of all even numbers and let $\mathbb{N}_{2}$ be the set of all odd numbers from $\mathbb{N}$. Define functions $f_{1}, f_{2}: X \rightarrow \mathbb{R}$ as follows:

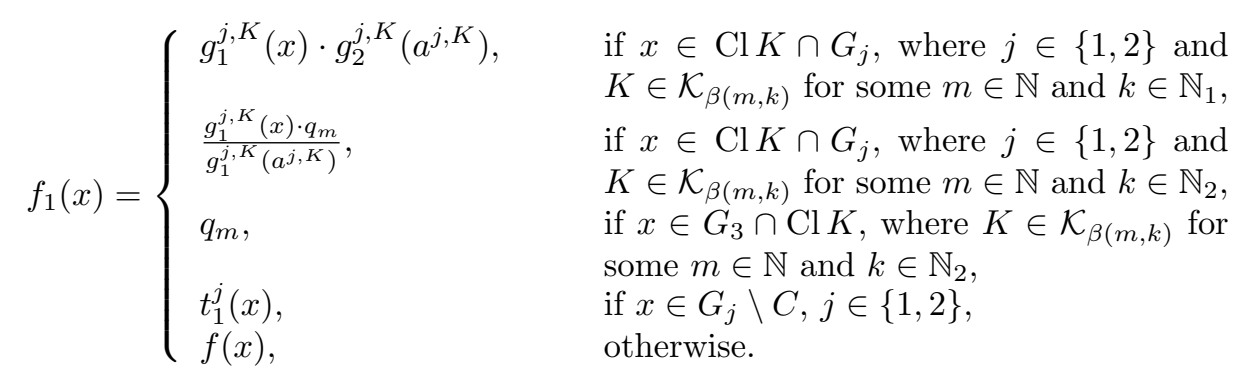




$$
f_{2}(x)= \begin{cases}\frac{g_{2}^{j, K}(x)}{g_{2}^{j, K}\left(a^{j, K}\right)}, & \text { if } x \in \mathrm{Cl} K \cap G_{j}, \text { where } j \in\{1,2\} \text { and } \\ \frac{g_{2}^{j, K}(x) \cdot g_{1}^{j, K}\left(a^{j, K}\right)}{q_{m}}, & K \in \mathcal{K}_{\beta(m, k)} \text { for some } m \in \mathbb{N} \text { and } k \in \mathbb{N}_{1}, \\ & K \in \mathcal{K}_{\beta(m, k)} \text { for some } m \in \mathbb{N} \text { and } k \in \mathbb{N}_{2}, \\ 0, & \text { if } x \in \mathrm{Cl} K \cap G_{3}, \text { where } K \in \mathcal{K}_{\beta(m, k)} \text { for } \\ & \text { some } m \in \mathbb{N} \text { and } k \in \mathbb{N}_{2}, \\ t_{2}^{j}(x), & \text { if } x \in G_{j} \backslash C, j \in\{1,2\}, \\ 1, & \text { otherwise. }\end{cases}
$$

Then evidently $f=f_{1} \cdot f_{2}$. We shall show that $f_{1}$ and $f_{2}$ are quasicontinuous. Let $x \in A$, let $U$ be a neighborhood of $x$ and $\varepsilon>0$. Choose $m \in \mathbb{N}$ such that $\left|q_{m}-f(x)\right|<\varepsilon / 2$. By (iii) there is $k \in \mathbb{N}_{2}$ and $K \in \mathcal{K}_{\beta(m, k)}$ such that $\mathrm{Cl} K \subset U$. By (i), $\mathrm{Cl} K \subset G_{1} \cup G_{2} \cup G_{3}$.

If $\mathrm{Cl} K \cap G_{3} \neq \emptyset$, then $G=K \cap G_{3} \subset U$ is a nonempty open set and $\left|f_{1}(x)-f_{1}(y)\right|=\left|f(x)-q_{m}\right|<\varepsilon$ for each $y \in G$.

If $\mathrm{Cl} K \cap G_{j} \neq \emptyset$, where $j \in\{1,2\}$, then $H=K \cap G_{j} \subset U$ is an open nonempty set. Since $g_{1}^{j, K}$ is quasicontinuous at $a^{j, K}$ there is an open nonempty set $G \subset H$ such that $\left|g_{1}^{j, K}(y)-g_{1}^{j, K}\left(a^{j, K}\right)\right|<\frac{\varepsilon}{2\left|q_{m}\right|} \cdot\left|g_{1}^{j, K}\left(a^{j, K}\right)\right|$ for each $y \in G$. Therefore for each $y \in G$ we have

$\left|f_{1}(y)-f_{1}(x)\right| \leq\left|f_{1}(y)-f_{1}\left(a^{j, K}\right)\right|+\left|f_{1}\left(a^{j, K}\right)-f_{1}(x)\right|<\varepsilon / 2+\left|q_{m}-f(x)\right|<\varepsilon$.

Further, there is $l \in \mathbb{N}_{1}$ and $L \in \mathcal{K}_{\beta(m, l)}$ such that $\mathrm{Cl} L \subset U$. If $\mathrm{Cl} L \cap G_{3} \neq$ $\emptyset$, then $G=K \cap G_{3} \subset U$ is an open nonempty set and $\left|f_{2}(y)-f_{2}(x)\right|=0$ for each $y \in G$.

If $\mathrm{Cl} L \cap G_{j} \neq \emptyset$, where $j \in\{1,2\}$, then there is an open nonempty set $G \subset K \cap G_{j} \subset U$ such that for each $y \in G$ we have $\left|g_{2}^{j, L}(y)-g_{2}^{j, L}\left(a^{j, L}\right)\right|<$ $\varepsilon\left|g_{2}^{j, L}\left(a^{j, L}\right)\right|$ and therefore $\left|f_{2}(y)-f_{2}(x)\right| \leq\left|f_{2}(y)-f_{2}\left(a^{j, L}\right)\right|+\mid f_{2}\left(a^{j, L}\right)-$ $f_{2}(x) \mid<\varepsilon$. Therefore $f_{1}$ and $f_{2}$ are quasicontinuous at each $x \in A$. The quasicontinuity at the other points is easy to see. If $f$ is in Baire class $\alpha$ then also $f_{1}$ and $f_{2}$ are in Baire class $\alpha$.

Remark 3.5. There is a normal (not $T_{1}$ ) second countable space $X$ such that every quasicontinuous function defined on $X$ is constant but there are nonconstant functions from $\mathcal{H}$ [2] .

Remark 3.6. In [2] it is shown that if $X$ is a Baire second countable space such that the family of all open connected sets is a $\pi$-base for $X$, then every function from $\mathcal{H}$ is the product of two simply continuous functions. It follows 
from Theorem 3.4 that this result is true for a pseudometrizable space $X$ which gives a positive answer to Problem 1 in [2] (if $X$ is pseudometrizable then we have $\mathcal{H} \subset \mathcal{S} \cdot \mathcal{S}$ and if $X$ is moreover Baire, then $\mathcal{H}=\mathcal{S} \cdot \mathcal{S})$.

Remark 3.7. In Theorem 3.4 we cannot assure $f_{1}$ and $f_{2}$ to be bounded if $f \in \mathcal{H}$ is bounded. Theorem V.2.2 in [9] gives a characterization of functions $f: \mathbb{R} \rightarrow \mathbb{R}$ which can be written as the product of $k$ bounded quasicontinuous functions. From this characterization it follows that e. g. the characteristic

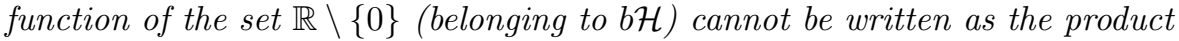
of finitely many bounded quasicontinuous functions. It is a natural problem to characterize functions $f: X \rightarrow \mathbb{R}$ which can be written as the product of $k$ bounded quasicontinuous functions for a pseudometrizable space $X$.

In [6] it is shown that if $f: \mathbb{R} \rightarrow \mathbb{R}$ is cliquish then there are $c \in \mathbb{R}$ and quasicontinuous functions $f_{1}, f_{2}: \mathbb{R} \rightarrow \mathbb{R}$ such that $f=c+f_{1} f_{2}$. We shall show that this result can be generalized to a separable pseudometrizable space but not to an arbitrary pseudometrizable space.

Theorem 3.8. Let $X$ be a pseudometrizable space whose weight is less than the cardinality of $\mathbb{R}$. Then for each cliquish function $f: X \rightarrow \mathbb{R}$ there are $c \in \mathbb{R}$ and quasicontinuous functions $f_{1}, f_{2}: X \rightarrow \mathbb{R}$ such that $f=c+f_{1} f_{2}$.

Proof. Let $A=\left\{a \in \mathbb{R}: f^{-1}((a, \infty))\right.$ is not simply open $\}$. If $A_{a}=$ $f^{-1}((a, \infty))$ is not simply open, then the set $A_{a} \backslash \operatorname{Int} A_{a}$ is not nowhere dense and there is an open set $J_{a}$ in $X$ such that $A_{a}$ and $X \backslash A_{a}$ are dense in $J_{a}$.

Assume that $J_{a} \cap J_{b} \neq \emptyset$ for some $a, b \in A, a<b$. Then the sets $A_{b}=$ $\{x \in X: f(x)>b\}$ and $X \backslash A_{a}=\{x \in X: f(x) \leq a\}$ are dense in $J_{a} \cap J_{b}$, a contradiction with the cliquishness of $f$. Since the weight of $X$ is less than the cardinality of $\mathbb{R}$ the cardinality of the set $A$ is less than the cardinality of $\mathbb{R}$. Similarly the cardinality of the set $\left\{a \in \mathbb{R}: f^{-1}((-\infty, a))\right.$ is not simply open $\}$ is less than the cardinality of $\mathbb{R}$. Therefore there is $c \in \mathbb{R}$ such that the sets $f^{-1}((-\infty, c))$ and $f^{-1}((c, \infty))$ are simply open. Now the function $f-c$ belongs to $\mathcal{H}$ and we apply Theorem 3.4 .

Example 3.9. Let $X=I \times \mathbb{R}$ (where $I=[0,1]$ ) with the metric $d$, where $d(x, y)=d\left(\left(x_{1}, x_{2}\right),\left(y_{1}, y_{2}\right)\right)=\max \left\{\left|x_{1}-y_{1}\right|, \operatorname{sign}\left|y_{1}-y_{2}\right|\right\}$. Let $g: I \rightarrow \mathbb{R}$ be the Riemann function (i.e. $g(z)=1 / q$, if $z=p / q$, where $p$, $q$ are relatively prime, $q>0$ and $g(z)=0$ otherwise). Define a function $f: X \rightarrow \mathbb{R}$ by

$$
f(x)=g\left(x_{1}\right)+x_{2} \quad \text { for } x=\left(x_{1}, x_{2}\right) .
$$

Then $f$ is cliquish function and $f \neq c+f_{1} f_{2}$ for each $c \in \mathbb{R}$ and $f_{1}, f_{2} \in \mathcal{Q}$. 


\section{References}

[1] N. Biswas, On some mappings in topological spaces, Bull. Calcutta Math. Soc. 61 (1969), 127-135.

[2] J. Borsík, Products of simply continuous and quasicontinuous functions, Math. Slovaca 45 (1995), 445-452.

[3] J. Borsík, Algebraic structures generated by real quasicontinuous functions, Tatra Mt. Math. Publ. 8, 175-184.

[4] J. Borsík, Sums of quasicontinuous functions for nonseparable spaces, Real Analysis Exch. 22 (1996-97), 328-337.

[5] Z. Grande, Sur les fonctions cliquish, Časopis Pěst. Mat. 110 (1985), $225-236$.

[6] Z. Grande, On some representations of a. e. continuous functions, Real Analysis Exch. 21 (1995-96), 175-180.

[7] A. Maliszewski, On the sums and the products of quasi-continuous functions, Real Analysis Exch. 20 (1994-95), 418-421.

[8] A. Maliszewski, Sums and products of quasicontinuous functions, Real Analysis Exch. 21 (1995-96), 320-329.

[9] A. Maliszewski, Darboux Property and Quasi-Continuity. A Uniform Approach, WSP, Slupsk, 1996.

[10] T. Natkaniec, Products of quasi-continuous functions, Math. Slovaca 40 (1990), 401-405.

[11] T. Neubrunn, Quasi-continuity, Real Analysis Exch. 14 (1988-89), 259306. 